\title{
ANÁLISE DA PRODUÇÃO CIENTÍFICA NOS CURSOS TÉCNICO DE NÍVEL MÉDIO DO IFRN CAMPUS PAU DOS FERROS: ESTUDO DE CASO DAS TURMAS 2012.1 (2012- 2015)
}

\author{
C. E. F. CRUZ*, E. A. SOUZA, R. C. D. N. RÊGO e A. B. F. AMORIM \\ Instituto Federal de Educação, Ciência e Tecnologia do Rio Grande do Norte \\ cefcruz12@yahoo.com.br
}

Artigo submetido em outubro/2015 e aceito em dezembro/2015

DOI: $10.15628 /$ rbept.2015.3548

\section{RESUMO}

O presente trabalho configura-se como resultado de discussões sobre a iniciação científica no ensino técnico, e suas implicações no processo de produção cientifica por meio do contato dos alunos com a reunião e análise dados e vivência da escrita acadêmica de projetos e artigos científicos. Nesse contexto, a pesquisa objetivouse analisar a produção técnica-científica dos cursos técnicos de nível médio do Campus Pau dos Ferros do IFRN, no período de 2012 a 2015. Para realização do estudo, foram aplicados os métodos de pesquisas bibliográfica, qualitativa e de levantamento com 97 alunos dos cursos de Alimentos, Apicultura e Informática.
Constatou-se que $38,1 \%$ dos alunos participaram de projetos de pesquisa e/ou extensão; $61,9 \%$ participaram de eventos científicos; $44,3 \%$ publicaram trabalhos em periódicos e/ou eventos científicos; 39,2\% submeteram trabalhos para periódicos e/ou eventos científicos; por fim, que $52,6 \%$ dos alunos atuaram em onze áreas temáticas diferentes. Conclui-se que a iniciação científica se apresentou como um importante instrumento didático-pedagógico para o contato e experimentação dos alunos com a pesquisa acadêmica desde o ensino médio.

PALAVRAS-CHAVE: Diagnóstico escolar, Ensino Médio, Iniciação científica

\section{ANALYSIS OF SCIENTIFIC PRODUCTION IN THE COURSE OF TECHNICAL MIDDLE LEVEL OF THE CAMPUS PAU DOS FERROS - IFRN: CASE STUDY OF CLASSES 2012.1 (2012-2015)}

\begin{abstract}
This work presents the result of discussions on scientific research in technical education, and its implications in the scientific production process through contact of students with the meeting and analyzing data and experience of academic writing projects and scientific papers. In this context, the research aimed to analyze the technical-scientific production of mid-level technical courses Campus Pau dos Ferros IFRN in the period from 2012 to 2015. To conduct the study, methods of bibliographical research, qualitative and were applied survey with 97 students Food courses, Beekeeping and
\end{abstract}

Information Technology. It was found that $38.1 \%$ of students participated in research projects and / or extension; $61.9 \%$ participated in scientific events; $44.3 \%$ work published in journals and / or scientific events; $39.2 \%$ submitted papers to journals and / or scientific events; finally, that $52.6 \%$ of students were active in eleven different subject areas. It is concluded that scientific initiation is presented as an important didactic and pedagogical tool for contact and experimentation of students with academic research since high school.

KEYWORDS: Diagnostic school, High School, Scientific Initiation 


\section{INTRODUÇÃO}

Para Pereira (2003), estamos situados em uma era em que a ciência, a pesquisa e o desenvolvimento tecnológico constituem itens estratégicos para aumentar a competividade de um país baseada em produção de conhecimento e melhorar a qualidade de vida de sua população. A instauração de centros federais de educação tecnológica como locais postos na condição de propulsores do desenvolvimento local, é o elemento indispensável a constituição de um comprometimento dessas instituições com pesquisa e desenvolvimento (PEREIRA, 2003).

Os Institutos Federais (IFs) caracterizam-se por uma estrutura multicampi apresentando uma proposta de autonomia administrativa e pedagógica, no que garante, como sua finalidade acadêmica, o mínimo de $50 \%$ de vagas para cursos técnicos de nível médio, bem como o mínimo de $20 \%$ para cursos de licenciatura e/ou programas de formação de professores para a educação básica (FERNANDES, 2008).

Em setembro de 2009 com o início das atividades do Campus do IFRN sediado no município de Pau dos Ferros, toda a região do Alto Oeste Potiguar passou a contar com um espaço que objetiva formar profissionais-cidadãs alinhados as esferas do trabalho, ciência, tecnologia e cultura. A construção do Instituto no interior do Rio Grande do Norte integrou a segunda fase do Plano de Expansão da Rede Federal de Educação Profissional e Tecnológica do Ministério da Educação, lançada em 2007 com o objetivo de entregar até 2010 mais 150 unidades, totalizando 354 instituições em todas as regiões do país.

As práticas educativas nos IFs, ao trabalhar na tentativa de romper a fragmentação secular que existiu entre ciência/tecnologia e teoria/prática na pesquisa como princípio pedagógico e nas ações de extensão como forma de diálogo com a sociedade, revela-nos um formato novo de trabalhar com o conhecimento de maneira interdisciplinar (PACHECO, 2011).

Atribuído como instrumento indispensável à prática investigadora no sistema educacional, Reali (2011) definirá diagnóstico escolar como sendo "a reunião, interpretação e encaminhamentos de dados relevantes da vida pedagógica do estudante". Este método de avaliação escolar pode ser realizado a partir do levantamento de dados provenientes de questionários, entrevistas, relatos, observações, análise documental, diário de campo, etc. Tendo como função final a pretensão de melhorar o processo de ensino-aprendizagem, a produção científica, nesse aspecto, mostra-se eficaz no apontamento de orientações úteis a construção de um diagnóstico em sala de aula (REALI, 2011).

Nessa perspectiva, o presente artigo objetivou avaliar a produção científica nos cursos técnicos de nível médio em Alimentos, Apicultura e Informática do Instituto Federal de Educação, Ciência e Tecnologia do Rio Grande do Norte (IFRN), Campus de Pau dos Ferros, no período de 2012 a 2015.

\section{REVISÃO DE LITERATURA}

O Ministério da Educação no ano de 2003 organizou dois seminários nacionais de Educação Profissional, respectivamente, tematizados como: "Ensino Médio: Construção Política" e "Concepções, experiências, problemas e propostas". Estes dois momentos foram responsáveis por principiar discussões sobre as finalidades do ensino médio técnico, na tentativa de abdicar de uma 
determinação histórica centrada no mercado de trabalho, permitindo a construção de uma política educacional que supere a dualidade entre formação técnica e formação propedêutica (BRASIL, 2007).

De acordo com a Lei no 11.892, que cria os Institutos Federais e institui a Rede Federal de Educação Profissional, Científica e Tecnológica, estes são caracterizados como estabelecimentos públicos responsabilizados em ofertar educação profissional e tecnológica em todos os níveis e modalidades, desde a formação inicial e continuada de trabalhadores, ensino médio técnico, graduações tecnológica, bacharelado e licenciatura e cursos de pós-graduação, visando à formação e qualificação de cidadãos destinados a atuação profissional no mercado de trabalho nos mais diversos setores da economia. Além disso, ainda objetiva promover a produção tecnológica, científica e cultural, consequentemente relacionada ao desenvolvimento socioeconômico local, regional e nacional (BRASIL, 2008).

A iniciação científica é uma atividade desenvolvida no Brasil desde a década de 1950, pela qual desafia e concede um conjunto de instrumentos e saberes indispensáveis a introdução de jovens no processo educativo da pesquisa acadêmica, instigando-os a produzir e expor seus conhecimentos (BIANCHETTI; SILVA; OLIVEIRA, 2012). Ainda para os autores, é nesta vivência que o estudante pode utilizar técnicas e conceitos oriundos de debates em sala de aula, ampliar e vivenciar seus conhecimentos e, assim, constituir-se pesquisadores.

A pesquisa como princípio pedagógico pode ser entendida como uma prática que "instiga o estudante no sentido da curiosidade em direção ao mundo que o cerca, gera inquietude, possibilitando que o estudante possa ser protagonista na busca de informações e de saberes, que sejam do senso comum, escolares ou científicos" (BRASIL, 2012; 2013). Ademais, é a partir do estímulo a iniciação cientifica no ambiente escolar, que possibilitamos identificar uma juventude criativa e, portanto, gerar uma sociedade socioeconomicamente desenvolvida (FAVA-DE-MORAIS e FAVA, 2000).

Em 1997, o Brasil conseguiu entrar no grupo dos vinte países mais produtores de ciência e tecnologia no mundo, embora esteja na posição de décimo oitavo (FAVA-DE-MORAIS e FAVA, 2000). De acordo com Baumgarten (2007), essa mudança originou-se após um novo direcionamento promovido pela gestão de ciência e tecnologia (CeT), definindo áreas e grupos estratégicos para a destinação de recursos escassos existentes no país. Estas medidas aspiravam um crescimento vertiginoso no setor de $\mathrm{CeT}$, tendo como ponto principal a reorganização do desenvolvimento científico e tecnológico brasileiro, condição fundamental para colocar o Brasil entre as nações mais competitivas do mundo no cenário econômico (BAUMGARTEN, 2007).

Segundo a Fapesp (2011), o Brasil representou, entre os anos de 2008 a 2010, 55,6\% das publicações de artigos científicos na América Latina, com um total de 94.622 trabalhos, tendo notáveis destaques as áreas de Agricultura (com 7.689 artigos), Química (7.484 artigos) e Física (6.929 artigos). As publicações brasileiras são $25,5 \%$ maiores que a soma das publicações da Argentina, Colômbia, Chile, México e Venezuela, com um total de 75.665 publicações, juntos (FAPESP, 2011). 


\section{MATERIAL E MÉTODOS}

A pesquisa foi desenvolvida no Campus de Pau dos Ferros do Instituto Federal de Educação, Ciência e Tecnologia do Rio Grande do Norte (IFRN), situado a cerca de 400 km de Natal, capital do Estado, numa área de $28.820 \mathrm{~km}^{2}$ as margens da BR 405, km 154, próximo ao bairro Chico Cajá, no município já descrito. O Campus, inaugurado em 2009, atende aproximadamente 30 municípios diferentes, incluindo alguns dos estados do Ceará e Paraíba, tendo nos quatro anos iniciais 1200 alunos matriculados em cursos regulares, e conta atualmente com 70 professores. 0 eixo tecnológico em Pau dos Ferros é referente ao setor de Informática e Serviços.

Para elaboração do presente estudo, foi empregada os métodos de pesquisas bibliográfica, qualitativa e de levantamento. O levantamento dos dados compreendeu o mês de maio de 2015, com a elaboração e aplicação de um questionário semiestruturado contendo questões dicotômicas, de múltipla escolha e abertas, aplicados com 97 alunos das turmas 2012.1 dos cursos técnicos de Alimentos, Apicultura e Informática, a fim de identificar a produção científica no ensino técnico durante o período de 2012 a 2015 por meio da participação em projetos de pesquisa e extensão, atuação em estágios, participação em eventos científicos, publicação de trabalhos em periódicos e eventos científicos, trabalhos submetidos para periódicos e/ou eventos científicos, temáticas abordadas nas pesquisas e trabalhos e, por fim, os motivos que levaram a inserção na produção científica. Do total de 97 entrevistas, 36 foram com os alunos da turma de Alimentos, 33 com os alunos de Apicultura, e 28 com a turma de Informática.

Em todas as entrevistas foram registradas as informações cadastrais de nome completo, idade, sexo, local e data. Para o cálculo estatístico e a elaboração dos gráficos contaram com o auxílio do software Microsoft Excel 15.0 (Office 2013).

\section{RESULTADOS E DISCUSSÃO}

Pela Figura 01 podemos observar que $24,7 \%$ dos estudantes dos cursos técnicos do IFRN de Pau dos Ferros participaram de algum projeto de pesquisa durante o período avaliado. Em um percentual menor, Posztbiegel et al. (2011) identificaram que a realização de pesquisas pelos cursos técnicos no Campus III do Centro Federal de Educação Tecnológica de Minas Gerais representa apenas $16 \%$ destas atividades, com um percentual de $0,8 \%$ de alunos destes cursos envolvidos. Para Grümm, Vieira e Brito (2013), a participação em projetos de pesquisa ou iniciação científica no ensino médio apresenta-se como um dos espaços que permite ao estudantepesquisador a experimentação do 'fazer ciência', proporcionando o desenvolvimento da autonomia tanto pela construção do conhecimento quanto pela resolução de problemas.

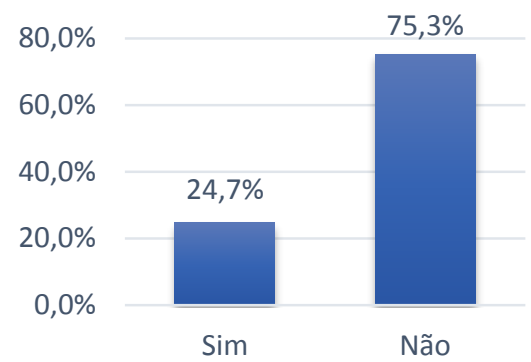

Figura 01 - Percentual de alunos que participaram de projetos de pesquisa no período 2012-2015. Fonte: Elaborado pelos autores do trabalho. 
A partir da análise da Figura 02, observamos que apenas 13,4\% dos alunos apresentaramse inclusos em algum projeto de extensão.

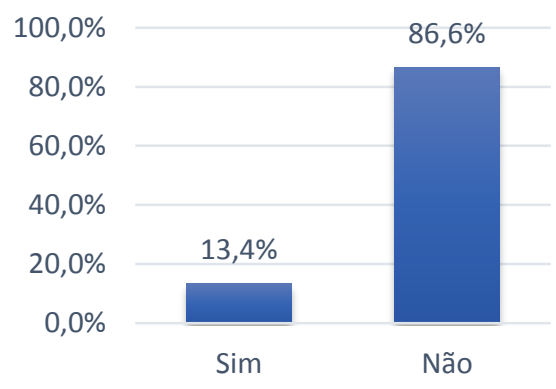

Figura 02 - Participação de estudantes em projetos de extensão.

Fonte: Elaborado pelos autores do trabalho.

Quanto ao estágio, o número representa somente 6,2\% dos entrevistados (FIGURA 03). Nesse aspecto, por tratar-se de prática profissional, analisando os números percebemos a quão pequena é a quantidade de estudantes inseridos nessa atividade, o que pode indicar o desinteresse pelo exercício prático do que foi trabalhado em sala ou, até mesmo, a falta de empresas nas áreas de Informática e Produção de Alimentos, tendo em vista os cursos ofertados no Campus de Pau dos Ferros.

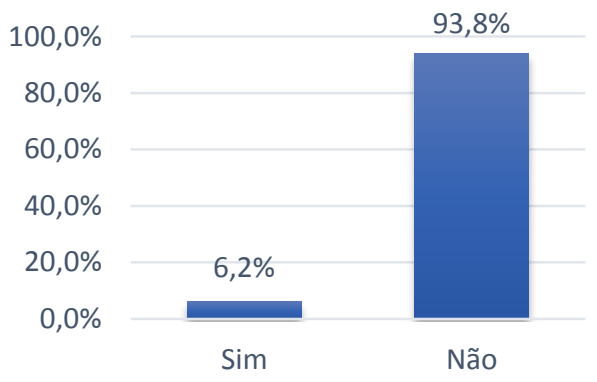

Figura 03 - Atuação dos alunos em estágios.

Fonte: Elaborado pelos autores do trabalho.

Referente a participação de alunos em eventos científicos, o percentual é de $61,9 \%$ dos alunos. Além disso, a média de participações em eventos é correspondente a 1,5 evento por aluno. Por ser fornecido ensino, pesquisa e extensão, o IFRN tem eventos próprios, como o Congresso de Iniciação Científica (CONGIC), além de sediar outros. Todas estas ações são para garantir a participação e contato dos alunos com pesquisadores, empresas e centros de pesquisas nacional e/ou internacional, despertando nestes o interesse pelo pensar cientificamente.

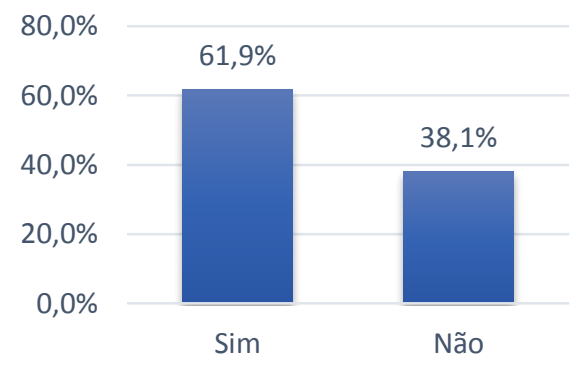

Figura 04 - Participação de alunos em eventos científicos no período de 2012-2015. Fonte: Elaborado pelos autores do trabalho. 
Na Figura 05, podemos visualizar que apenas 7,2\% dos estudantes publicaram trabalhos em periódicos científicos. Na pós-graduação, diferentemente do ensino técnico, a publicação de artigos em periódicos científicos nacionais e/ou internacionais torna-se importante para avaliação dos programas de pós-graduação das universidades, além de expor os pesquisadores e o país onde foi produzido como desenvolvedores de ciência e tecnologia.

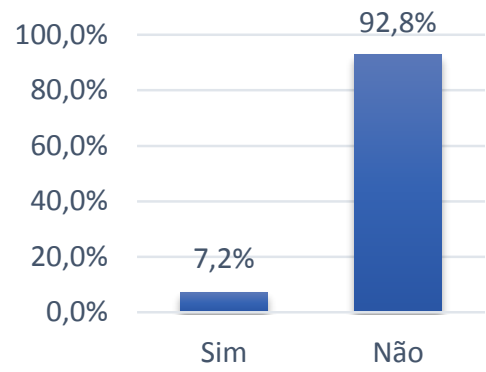

Figura 05 - Percentual de alunos que possuem publicações em periódicos científicos.

Fonte: Elaborado pelos autores do trabalho.

Referente as publicações em eventos científicos, na Figura 06, observa-se que $37,1 \%$ dos estudantes publicaram trabalhos em eventos científicos, durante o período de 2012 a 2015 . 0 percentual de publicações em eventos está, como podemos analisar, adequado com os valores de participação em projetos de pesquisa e extensão, evidenciando que as atividades práticas foram formalizadas em trabalhos técnico-científicos.

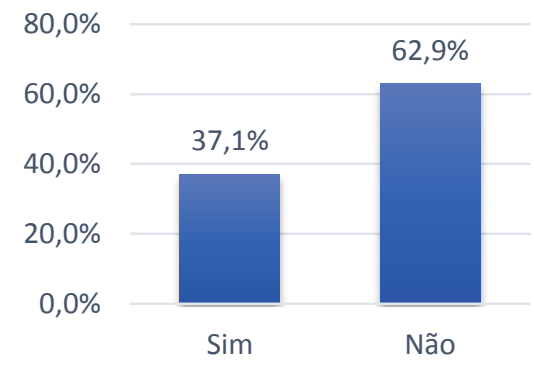

Figura 06 - Percentual de alunos que publicaram trabalhos em eventos científicos. Fonte: Elaborado pelos autores do trabalho.

Analisando a Figura 07, podemos notar o percentual de alunos que submeteram trabalhos para periódicos e/ou eventos científicos no ano de 2015 . Tendo em vista os $37,1 \%$ de alunos com trabalhos publicados em eventos científicos, a partir do dado seguinte percebemos um aumento na produção: $39,2 \%$ dos estudantes submeteram trabalhos resultados provenientes de projetos em execução ou de pesquisas pontuais nas mais diversas áreas.

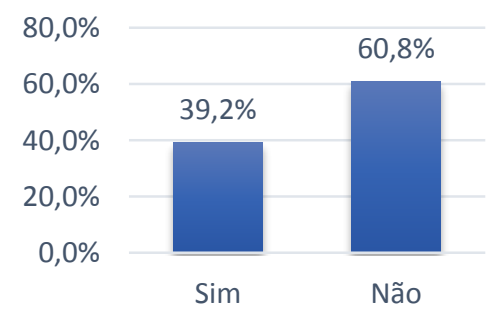

Figura 07 - Alunos que submeteram trabalhos para periódicos e/ou eventos científicos. Fonte: Elaborado pelos autores do trabalho. 
Com relação as áreas temáticas (ou linhas) de pesquisa, na Figura 08, podemos observar onze temáticas diferentes abordadas pelos professores e alunos no Campus de Pau dos Ferros do IFRN, dentre as quais destacam-se a Ciência e Tecnologia de Alimentos (20,6\%) e Apicultura, contando também com a participação de $20,6 \%$ dos estudantes entrevistados. Os dados indicam uma maior produção por partes dos professores e alunos das áreas técnicas, mostrando a pouca realização de pesquisas nas áreas propedêuticas, as de formação geral.

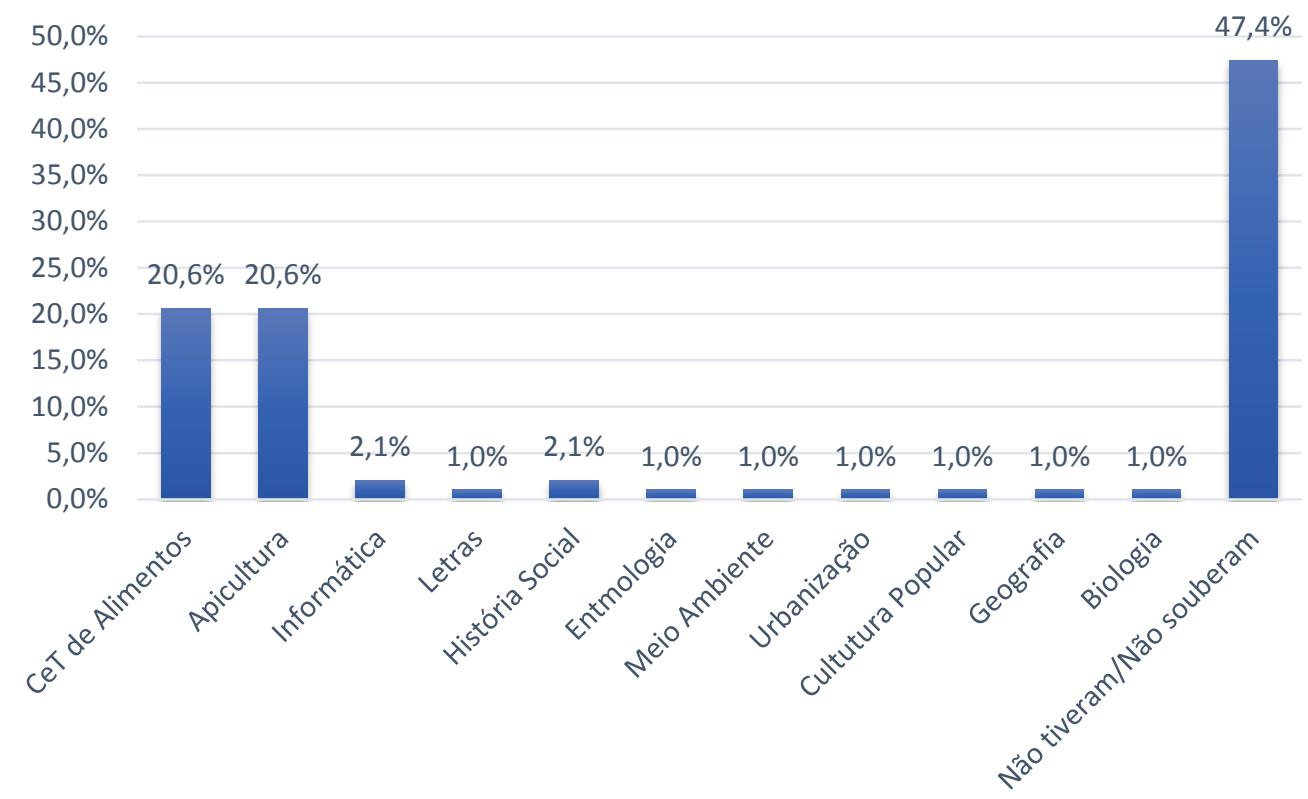

Figura 08 - Áreas temáticas abordadas nas pesquisas e trabalhos pelos alunos. Fonte: Elaborado pelos autores do trabalho.

Por último, foi analisado os motivos que levaram o ingresso dos estudantes a produção científica nos seus cursos do IFRN de Pau dos Ferros. Visualizando a Figura 09 podemos perceber que $24,7 \%$ dos estudantes tiveram uma iniciativa própria, principiando as suas pesquisas na instituição. Os alunos que foram incentivados pelos professores representam um percentual de $13,4 \%$. Os dados indicam que o incentivo dos professores $(13,4 \%)$ relacionado a construção de métodos de produção de projetos e artigos como requisito de avaliação em disciplinas $(3,1 \%)$ e a divulgação de congresso/seminários/simpósios/feiras pelo Campus de Pau dos Ferros, promovem, na maioria das vezes, interesses próprios $(24,7 \%)$ de vivência científica e intercâmbio de informações, que gradativamente vão sendo percebidos por outros professores e alunos, incentivando-os (3,1\%).

De modo equivalente ao uso do método de avaliação em disciplinas utilizados pelos professores como estímulo à produção cientifica, Souza et al. (2013) mostra que no curso Técnico em Apicultura, além da formação técnica direcionada ao manejo de abelhas sociais, semi-sociais e solitárias e da cadeia produtiva da apicultura no todo, há ações didático-pedagógicas por meio da criação da Comissão de Co-Orientação Científica em Apicultura, utilizando a pesquisa científica como instrumento para apreensão dos conceitos e desenvolvimento de habilidades e experiências para fixação de conhecimentos adquiridos nas aulas teórico-práticas. Ainda segundo o autor, durante sua implementação, entre os anos de 2012 e 2013, a comissão envolveu 45 alunos do curso na organização destas atividades de iniciação científica. 


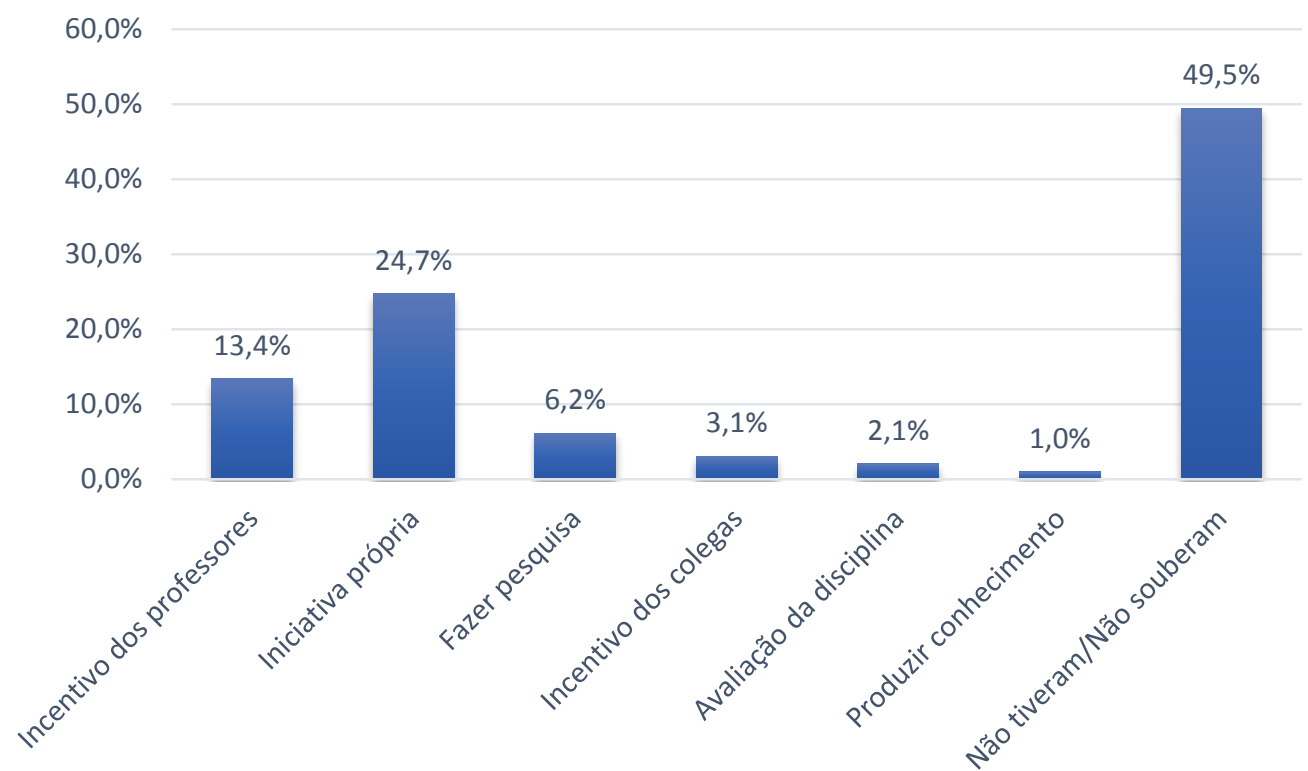

Figura 09 - Motivos apresentados pelos estudantes que resultaram no ingresso as produções científicas. Fonte: Elaborado pelos autores do trabalho.

\section{CONCLUSÃO}

Conclui-se que o ingresso dos estudantes na iniciação científica, a partir dos dados apresentados, mostrou-se como excelente ferramenta para o contato e vivência com a pesquisa acadêmica desde o ensino médio, notando uma participação de $52,6 \%$ de alunos em diferentes áreas temáticas executando atividades de pesquisa e extensão (bolsistas remunerado ou voluntário).

A participação, publicação e submissão de trabalhos em eventos científicos, além da diversidade de temáticas trabalhadas no IFRN de Pau dos Ferros, indicaram ações efetivas pelos professores e gestão do Campus, com o fito de proporcionar crescimento e desenvolvimento acadêmico dos estudantes e, consequentemente, contribuição para o progresso das áreas de estudo.

\section{REFERÊNCIAS BIBLIOGRÁFICAS}

1. BAUMGARTEN, M. Geopolítica do conhecimento e da informação: semiperiferia e estratégias de desenvolvimento. Liinc em Revista, Rio de Janeiro/RJ, v. 3, n. 1, p. 16-32, mar. 2007.

2. BIANCHETTI, L.; SILVA, E. L.; OLIVEIRA, A. A iniciação à pesquisa no Brasil: políticas de formação de jovens pesquisadores. In: SEMINÁRIO DE PESQUISA EM EDUCAÇÃO DA REGIÃO SUL, 9., 2012. Caxias do Sul/RS. Anais. Caxias do Sul/RS: UCS, 2012. Disponível em: < http://www.ucs.br/etc/conferencias/index.php/anpedsul/9anpedsul/paper/viewFile/633/72 7>. Acesso em: 27 de mai. 2015.

3. BRASIL. Ministério da Educação. Secretaria de Educação Básica. Secretaria de Educação Continuada, Alfabetização, Diversidade e Inclusão. Secretaria de Educação Profissional e Tecnológica. Conselho Nacional de Educação. Câmara Nacional de Educação Básica. Diretrizes Curriculares Nacionais Gerais da Educação Básica. Brasília/DF: MEC, SEB, DICEI, 2013. 592 p. 
4. Ministério da Educação. Secretaria Executiva. Secretaria de Educação Profissional e Tecnológica. Educação Profissional Técnica de Nível Médio Integrada ao Ensino Médio. Documento Base. Brasília/DF: MEC, 2007.

5. _ Ministério da Educação. Conselho Nacional de Educação. Câmara Nacional de Educação Básica. Resolução CNE/CEB no 6, de 20 de setembro de 2012. Dispõe sobre as Diretrizes Curriculares Nacionais para a Educação Profissional Técnica de Nível Médio. Diário Oficial da República Federativa do Brasil, Brasília/DF, n. 6, set. 2012. 12 p.

6. Presidência da República. Casa Civil. Subchefia para Assuntos Jurídicos. Lei no 11.892, de 29 de dezembro de 2008. Institui a Rede Federal de Educação Profissional, Científica e Tecnológica, cria os Institutos Federais de Educação, Ciência e Tecnologia, e dá outras providências. Diário Oficial da República Federativa do Brasil, Brasília/DF, dez. 2008.

7. FAPESP. Fundação de Amparo à Pesquisa do Estado de São Paulo. Pesquisadores brasileiros publicam $56 \%$ dos artigos científicos originados na América Latina. Boletim de Indicadores Fapesp de Ciência, Tecnologia e Inovação, n. 3, nov. 2011. Disponível em: < http://www.fapesp.br/indicadores/boletim3.pdf>. Acesso em: 27 de mai. 2015.

8. FAVA-DE-MORAIS, F.; FAVA, M. A iniciação científica: muitas vantagens e poucos riscos. São Paulo em Perspectiva, São Paulo/SP, v. 14, n. 1, jan./mar. 2000.

9. FERNANDES, F. C. M. Novo Design para a Rede Federal de Educação Tecnológica. Holos, Natal/RN, v. 3, p. 56-66, 2008.

10. GRÜMM, C. A. F.; VIEIRA, S. F.; BRITO, L. M. A iniciação científica no ensino médio integrado como possibilidade de uma prática integradora: estudo de caso através do resgaste da memória da vitivinicultura em Videira, Santa Catarina. In: COLÓQUIO NACIONAL - A PRODUÇÃO DO CONHECIMENTO EM EDUCAÇÃO PROFISSIONAL, 2., 2013. Natal/RN. Anais. Natal/RN: IFRN, 2013.

11. PACHECO, E. (Org.). Institutos Federais: uma revolução na educação profissional e tecnológica. São Paulo/SP: Editora Moderna, 2011.

12. PEREIRA, L. A. C. A Rede Federal de Educação Tecnológica e o Desenvolvimento Local. 2003. 114 f. Dissertação (Mestrado em Planejamento Regional e Gestão de Cidades) - Universidade Cândido Mendes. Campos dos Goytacazes/RJ, 2003.

13. POSZTBIEGEL, L.; MATOS, R. P.; TEMOTEO, A. S.; OLIVEIRA, A. R.; CARMO, M. J. Importância da iniciação científica para discentes de instituições de ensino técnico e superior: um estudo de caso de Campus III do CEFET-MG. In: CONGRESSO BRASILEIRO DE EDUCAÇÃO EM ENGENHARIA, 39., 2011. Blumenau/SC. Anais. Brasília/DF: ABENGE, 2011.

14. REALI, N. G. Diagnóstico escolar: implicações político-pedagógicas e questões metodológicas. In: COLÓQUIO INTERNACIONAL DE EDUCAÇÃO, 4., 2011. Joaçaba/SC. Anais. Joaçaba/SC: Editora Unoesc, v. 1, n. 1, 2011. Disponível em: < http://editora.unoesc.edu.br/index.php/coloquiointernacional/article/view/1267>. Acesso em: 26 de mai. 2015.

15. SOUZA, E. A.; PENHA, L. S.; QUEIROZ, G. S.; SILVA, A. C. R.; MESQUITA, L. X.; PEREIRA, D. S. O Pensar Cientificamente no Ensino Técnico: Comissão de Co-Orientação Científica em Apicultura - CCoCAp, no IFRN - Campus Pau Dos Ferros. In: SEMINÁRIO BRASILEIRO DE PRÓPOLIS E PÓLEN, 2., 2013. Ilhéus/BA. Anais. Cruz das Almas/BA: Magistra, 2013. 